\title{
Stepwise Hydrogen Atom and Proton Transfers in Dioxygen Reduction by Aryl-Alcohol Oxidase
}

\author{
Juan Carro, ${ }^{1}$ Patricia Ferreira, ${ }^{2,3}$ Angel T. Martínez ${ }^{* 1}$ and Giovanni Gadda*4,5,6,7 \\ ${ }^{1}$ Centro de Investigaciones Biológicas, CSIC, Ramiro de Maeztu 9, E-28040 Madrid, Spain \\ ${ }^{2}$ Departament of Biochemistry and Cellular and Molecular Biology, and ${ }^{3}$ Institute for \\ Biocomputation and Physics of Complex Systems (BIFI), University of Zaragoza, E-50009 \\ Zaragoza, Spain
}

${ }^{4}$ Department of Chemistry, ${ }^{5}$ Department of Biology, ${ }^{6}$ Center for Biotechnology and Drug Design, and ${ }^{7}$ Center for Diagnostics and Therapeutics, Georgia State University, Atlanta, Georgia 303023965, USA

Juan Carro, E-mail: jcarro@cib.csic.es

Patricia Ferreira, E-mail: ferreira@unizar.es

Angel T. Martínez, E-mail: atmartinez@cib.csic.es

Giovanni Gadda, E-mail: ggadda@gsu.edu 
ABSTRACT: The mechanism of dioxygen reduction by the flavoenzyme aryl-alcohol oxidase was investigated with kinetic isotope, viscosity, and $\mathrm{pL}(\mathrm{pH} / \mathrm{pD})$ effects in rapid kinetics experiments by stopped-flow spectrophotometry of the oxidative half-reaction of the enzyme. Doublemixing of the enzyme in a stopped-flow spectrophotometer with $\left[\alpha-{ }^{2} \mathrm{H}_{2}\right]-p$-methoxybenzyl alcohol and oxygen at varying aging times established a slow rate constant of $0.0023 \mathrm{~s}^{-1}$ for the wash-out of the D atom from the N5 atom of the reduced flavin. Thus, the deuterated substrate could be used to probe the cleavage of the $\mathrm{N}-\mathrm{H}$ bond of the reduced flavin in the oxidative halfreaction. A significant and $\mathrm{pH}$-independent substrate $\mathrm{KIE}$ of 1.5 between $\mathrm{pH} 5.0$ and 8.0 demonstrated that $\mathrm{H}$ transfer is partially limiting the oxidative half-reaction of the enzyme; a negligible solvent KIE of 1.0 between PD 5.0 and 8.0 proved a fast $\mathrm{H}^{+}$transfer reaction that does not contribute to determining the flavin oxidation rates. Thus, a mechanism for dioxygen reduction in which the $\mathrm{H}$ atom originating from the reduced flavin and $\mathrm{a}^{+}$from a solvent exchangeable site are transferred in separate kinetic steps is proposed. The spectroscopic and kinetic data presented also showed lack of stabilization of transient flavin intermediates. The substantial differences in the mechanistic details of $\mathrm{O}_{2}$ reduction by aryl alcohol oxidase with respect to other alcohol oxidases like choline oxidase, pyranose 2-oxidase, and glucose oxidase, further demonstrates the high level of versatility of the flavin cofactor in flavoenzymes. 


\section{INTRODUCTION}

Reduction of $\mathrm{O}_{2}$ by flavoenzymes is a fascinating yet intriguing process in nature. ${ }^{1}$ It is an impaired reaction due to the intrinsic characteristics of the $\mathrm{O}_{2}$ and flavin molecules. Although the reaction is thermodynamically favored by the differences in redox potential between the oxygen and the flavin species participating in the reaction, ${ }^{2}$ given the difference in the spin ground state of the two molecules, with $\mathrm{O}_{2}$ in triplet state and flavin in singlet state, the reaction is impeded by the law of spin conservation. ${ }^{3}$ However, flavoprotein oxidases have overcome this restriction by single one-electron transfers in a stepwise manner. ${ }^{4}$ Flavin cofactors play a central role in the whole process as they act receiving two electrons from organic substrates during the reductive half-reaction and donating them to $\mathrm{O}_{2}$. The architecture of the enzyme active site is also important for the redox process, by allowing $\mathrm{O}_{2}$ access, activation, and reaction with the reduced flavin. In fact, flavoprotein oxidases speed up reactions with $\mathrm{O}_{2}$ by at least hundred-fold in comparison with free flavins aided by the presence of a positive charge and hydrophobic sites that localize $\mathrm{O}_{2}$ for the reaction. ${ }^{1}$

Flavoprotein oxidases initiate reduced flavin oxidation by transferring an electron from the hydroquinone flavin to $\mathrm{O}_{2}$, thereby producing a caged radical pair of a neutral semiquinone and the superoxide radical. ${ }^{1}$ The radical pair is highly unstable and decays with formation of hydrogen peroxide and oxidized flavin. ${ }^{5}$ Although the flavin semiquinone is usually not observed in oxidation reactions catalyzed by flavoprotein oxidases, an intermediate with features of a flavosemiquinone was detected with human liver glycolate oxidase. ${ }^{6}$ The first electron transfer from the hydroquinone to $\mathrm{O}_{2}$ has been shown to be rate-limiting in glucose oxidase. $^{7}$ Therefore, stabilization of the transition state to form the caged radical pair is crucial in these enzymes to attain the magnitude of acceleration of this first electron transfer. ${ }^{8,9}$ In contrast to monooxygenases, typically oxidases do not stabilize C4a-peroxyflavin intermediates after the second electron transfer and the decay of this radical pair. Two notable exceptions are glucose oxidase, in which pulse radiolysis data suggested the presence of the C4a intermediate, ${ }^{10}$ and pyranose 2-oxidase (P2O), in which a C4a-peroxyflavin was detected spectroscopically in the oxidative half-reaction. ${ }^{11,12}$ The reduction of $\mathrm{O}_{2}$ to $\mathrm{H}_{2} \mathrm{O}_{2}$ requires the transfer of an electron and a hydrogen atom from the reduced flavin to $\mathrm{O}_{2}$ and a proton from 
the solvent or a solvent exchangeable side chain in the active site of the enzyme. ${ }^{5}$ To date, the relative timing for the transfer of the hydrogen atom and proton has been elucidated by using kinetic isotope effects in a limited number of flavoproteins oxidase, including choline oxidase ${ }^{13}$ and $\mathrm{P} 2 \mathrm{O}^{12}$

The mechanisms underlying the oxygen reduction by aryl-alcohol oxidase (AAO; EC 1.1.3.7) from the basidiomycete Pleurotus eryngii has yet to be investigated. Several reports on the mechanism of action of AAO have been published, including the oxidation of alcohols ${ }^{14-16}$ and aldehydes $^{17}$, as well as structural-functional relationships of its oxidative half-reaction ${ }^{18-20}$. The physiological role of $A A O$ is to supply $\mathrm{H}_{2} \mathrm{O}_{2}$ that triggers Fenton reactions or fuels peroxidases in order to depolymerize lignocellulose. Therefore, production of $\mathrm{H}_{2} \mathrm{O}_{2}$ is an important feature of $A A O$, which encouraged its discovery and investigation ${ }^{21}$, and permitted its exploitation for the production of chemicals in enzyme cascades along with an enzyme whose oxidizing substrate is $\mathrm{H}_{2} \mathrm{O}_{2}{ }^{22}$ To the best of our knowledge, no other electron acceptor has been found for AAO from P. eryngii so far, opposite to what has been seen for other GMC oxidoreductases as glucose oxidase $^{23}$ and the recently-described quinone-dependent aryl-alcohol dehydrogenases. ${ }^{24}$

In the work presented here, we have further advanced the mechanistic knowledge on $P$. eryngii $\mathrm{AAO}$ by investigating the relative timing for hydrogen and proton transfer in the oxidative half-reaction catalyzed by the enzyme. Toward this end, we have used mechanistic probes, such as solvent, substrate, and multiple deuterium kinetic isotope effects (KIE), solvent viscosity and $\mathrm{pL}$ effects, with rapid reaction studies of the oxidative half-reaction catalyzed by AAO in a stopped-flow spectrophotometer. The results obtained provide a detailed mechanistic understanding of the oxidative half-reaction catalyzed by AAO and, when combined with the cases previously presented for choline oxidase and P2O, allow for further insights on the reaction catalyzed by flavoprotein oxidases as a class of oxidizing enzymes and the versatility of the flavin as cofactor. 


\section{MATERIALS AND METHODS}

Reagents. $p$-Methoxybenzyl alcohol ( $\geq 98 \%)$ and sodium deuteroxide (NaOD) $(99.5 \%$ isotopic purity) were purchased from Sigma-Aldrich (St. Louis, MO, USA). [ $\left.\alpha{ }^{2} \mathrm{H}_{2}\right]-p$-Methoxybenzyl alcohol was synthesized at the Instituto de Ciencia de Materiales de Aragón (Zaragoza, Spain). Deuterium oxide ( $\mathrm{D}_{2} \mathrm{O} ; 99.9 \%$ isotopic purity) was bought from Cambridge Isotope Co. (Andover, MA, USA).

Enzyme production and purification. AAO from $P$. eryngii (GenBank accession number AF064069) was heterologously expressed in E. coli W3110 as inclusion bodies and in vitro activated and purified as previously described. ${ }^{25}$

Spectroscopic studies. UV-visible absorption spectra were recorded in $50 \mathrm{mM}$ phosphate, $\mathrm{pH} 6.0$, at $25^{\circ} \mathrm{C}$, using an Agilent Technologies (Santa Clara, CA, USA) diode-array HP-8453 UVvisible spectrophotometer. To calculate enzyme concentration, the absorbance at $463 \mathrm{~nm}$ was used, together with the molar absorption coefficient $\left(\varepsilon_{463}=11,050 \mathrm{M}^{-1} \cdot \mathrm{cm}^{-1}\right)$ previously calculated by thermal denaturation and estimation of the free FAD release. ${ }^{25}$

Rapid kinetics. They were carried out on a TgK Scientific (Salisbury, UK) Model SF-61DX stopped-flow spectrophotometer in both double-mixing and-single mixing modes at $12^{\circ} \mathrm{C}$, unless otherwise stated.

The enzyme was prepared to use by gel filtration through a PD-10 desalting column (General Electric, Fairfield, CT), followed by centrifugation to remove denatured protein. The column was equilibrated with the desired buffer (prepared in $\mathrm{H}_{2} \mathrm{O}$ or $\mathrm{D}_{2} \mathrm{O}$, or containing glycerol), which was also used to elute the enzyme. Deuterated buffers were prepared by dissolving the salts in $\mathrm{D}_{2} \mathrm{O}$ and $\mathrm{pD}$ was adjusted with $\mathrm{NaOD}$ using a $\mathrm{pH}$-meter $(\mathrm{pD}=\mathrm{pH}$ reading +0.41$)$. Viscosity effect experiments were performed in $50 \mathrm{mM} \mathrm{Na}$ phosphate buffer, $\mathrm{pH}$ 6.0, containing 7.4\% (v:v) glycerol in order to mimic $\mathrm{D}_{2} \mathrm{O}$ viscosity at $12^{\circ} \mathrm{C}\left(1.6 \times 10^{-3} \mathrm{~N} \cdot \mathrm{s} \cdot \mathrm{m}^{-2}\right) .^{26}$

Enzyme kinetics were measured by monitoring the absorbance changes at $463 \mathrm{~nm}$, both in $\mathrm{H}_{2} \mathrm{O}$ and $\mathrm{D}_{2} \mathrm{O}$, at $12{ }^{\circ} \mathrm{C}$, unless otherwise stated. The enzyme solution, containing $1 \mathrm{mM}$ glucose, in either phosphate buffers, pL $6.0(50 \mathrm{mM}), 7.0(30 \mathrm{mM})$ or $8.0(25 \mathrm{mM})$, or $100 \mathrm{mM}$ acetate $\mathrm{pL}$ 5.0 was poured into the body of a tonometer, along with $0.05 \mu \mathrm{M}$ glucose oxidase, which was loaded into the tonometer's side arm. The enzyme was then subjected to 20-25 cycles of 
degassing by alternately applying vacuum and flushing with oxygen-free argon (pretreated with an oxygen scrubbing cartridge; Agilent, Palo Alto, CA). After a number of degassing cycles, the enzyme containing glucose was mixed with glucose oxidase in order to ensure anaerobiosis. Then, the degassed enzyme solution was mounted onto the stopped-flow equipment, which had previously been made anaerobic by an overnight treatment with $2 \mathrm{mM}$ glucose and $5 \mu \mathrm{M}$ glucose oxidase. The reducing substrates ( $p$-methoxybenzyl and $\left[\alpha-{ }^{2} \mathrm{H}_{2}\right]-p$-methoxybenzyl alcohols) were dissolved in the appropriate buffer and subsequently degassed by flushing oxygen-free $\operatorname{Ar}$ for at least $15 \mathrm{~min}$ prior to being mounted onto the stopped-flow spectrophotometer.

Enzyme reduction was measured by anaerobically mixing equal volumes of protein solution and organic substrate, yielding a reaction mixture containing $\sim 10 \mu \mathrm{M}$ AAO and $200 \mu \mathrm{M}$ of the organic substrate in $50 \mathrm{mM}$ Na phosphate, $\mathrm{pL} 6.0$.

Enzyme oxidation was measured by anaerobically mixing equal volumes of protein solution and organic substrate (0.9-fold the enzyme's concentration), which aged inside a loop until reaching complete reduction, before being mixed with an equal volume of buffer containing different $\mathrm{O}_{2}$ concentrations. This yielded a solution containing $\sim 5 \mu \mathrm{M}$ reduced $\mathrm{AAO}$ and $\mathrm{O}_{2}$ $(100-260 \mu \mathrm{M})$ in the desired buffer. Buffers containing $\mathrm{O}_{2}$ at various concentrations were prepared by bubbling different $\mathrm{O}_{2} / \mathrm{N}_{2}$ mixtures for $15 \mathrm{~min}$ in air-tight syringes. Actual $\mathrm{O}_{2}$ concentrations were measured at $25^{\circ} \mathrm{C}$ using a computer-interfaced Oxy-32 $\mathrm{O}_{2}$-monitoring system (Hansatech Instruments, Inc., Norfolk, England) just prior to the use of the buffers.

Data analysis. Kinetic data were fitted using the Kinetic Studio Software Suite (Hi-TgK Scientific, Bath, UK) and the KaleidaGraph software package (Synergy Software, Reading, PA). Stoppedflow traces fitted either Eq. 1 or Eq. 2, which describe a double exponential and single exponential processes, respectively, where $k_{\mathrm{obs} 1}$ and $k_{\mathrm{obs} 2}$ are the observed rate constants of the phases; $\mathrm{t}$ is time, $A$ is the absorption at $463 \mathrm{~nm}$ at any given time, $B_{1}$ and $B_{2}$ are the amplitude of absorption changes for the phases, and $C$ is the absorption at infinite time that accounts for the absorbance of the oxidized enzyme.

$$
\mathrm{A}_{463}=\mathrm{B}_{1} \mathrm{e}^{-k_{\text {obs } 1} \cdot \mathrm{t}}+\mathrm{B}_{2} \cdot \mathrm{e}^{-k_{\text {obs } 2} \cdot \mathrm{t}}+\mathrm{C}
$$




$$
\mathrm{A}_{463}=\mathrm{B}_{1} \mathrm{e}^{-k_{\mathrm{obs} 1} \cdot \mathrm{t}}+\mathrm{C}
$$

Rate constants were obtained by nonlinear fitting of the $k_{\mathrm{obs}}$ (obtained from averaging triplicates), at various $\mathrm{O}_{2}$ concentrations, to eq 2:

$$
k_{\mathrm{obs}}=k_{\mathrm{ox}} \cdot\left[\mathrm{O}_{2}\right]+k_{\mathrm{rev}}
$$

where $k_{\text {obs }}$ is the experimentally observed rate constant associated to flavin oxidation at any given substrate concentration, $k_{\text {rev }}$ is the reversible reaction, obtained as the intercept at $y$-axis; and $k_{\mathrm{ox}}$ is the second-order rate constant for the oxidative half-reaction. 


\section{RESULTS}

Aging Time for Double-Mixing Stopped-Flow Spectrophotometry. The reduced enzyme was prepared in a stopped-flow spectrophotometer by mixing anaerobically $20 \mu \mathrm{M}$ AAO with 0.9 molar equivalents of $\left[\alpha-{ }^{1} \mathrm{H}_{2}\right]-p$-methoxybenzyl alcohol at $\mathrm{pH} 6.0$ and $12{ }^{\circ} \mathrm{C}$. Enzyme reduction was followed at $463 \mathrm{~nm}$, establishing that the enzyme reached full reduction to hydroquinone within $1 \mathrm{~s}$ of mixing with the reducing substrate. Replacement of $\mathrm{H}_{2} \mathrm{O}$ with $\mathrm{D}_{2} \mathrm{O}$ yielded the same results. With $\left[\alpha-{ }^{2} \mathrm{H}_{2}\right]-p$-methoxybenzyl alcohol as a reducing substrate, full reduction was achieved in $3 \mathrm{~s}$ in $\mathrm{H}_{2} \mathrm{O}$ and $4 \mathrm{~s}$ in $\mathrm{D}_{2} \mathrm{O}$. These data establish the aging times to be used in double-mixing stopped-flow experiments for the investigation of the oxidative halfreaction of AAO.

Oxidative Half-Reaction vs. pH. The oxidative half-reaction was studied in a double-mixing stopped-flow spectrophotometer upon mixing the reduced enzyme prepared by aging AAO after anaerobic mixing with $\left[\alpha-{ }^{1} \mathrm{H}_{2}\right]-p$-methoxybenzyl alcohol (see above) with varying concentrations of molecular oxygen at $12{ }^{\circ} \mathrm{C}$. Flavin oxidation was monitored by following the increase in absorbance at $463 \mathrm{~nm}$ in PMT (single wavelength detection) mode, or with PDA detection to acquire time courses between $300 \mathrm{~nm}$ and $700 \mathrm{~nm}$. At pH 5.0 and $\mathrm{pH}$ 7.0, the increase in absorbance at $463 \mathrm{~nm}$ followed a monophasic pattern, as shown in Figure 1.

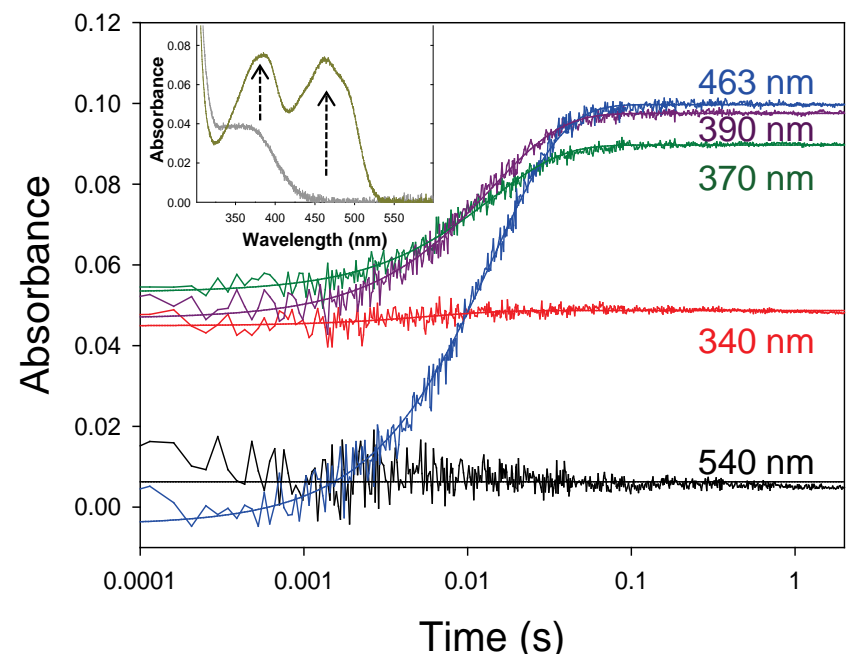

Figure 1. Time-resolved absorption spectroscopy of the oxidation of reduced AAO with $\mathrm{O}_{2}$ at several wavelengths. The anaerobic enzyme was premixed with $\left[\alpha-{ }^{1} \mathrm{H}_{2}\right]-p$-methoxybenzyl alcohol (0.9-fold the enzyme's concentration), allowed to age until complete flavin reduction was attained, and mixed with $140 \mu \mathrm{M} \mathrm{O}_{2}$ in a double-mixing stopped-flow spectrophotometer equipped with PDA detection. Conditions: enzyme ( $5 \mu \mathrm{M}$ after double mixing) in 
$100 \mathrm{mM}$ sodium phosphate, $\mathrm{pH} 5.0$, and $12^{\circ} \mathrm{C}$. Representative traces showing the oxidation of $\mathrm{AAO}$ by $\mathrm{O}_{2}$ at selected wavelengths characteristic of possible intermediates in the oxidative reaction. Inset shows the spectra of the reduced (grey) and oxidized (dark yellow) species of the enzyme with arrows indicating the direction of the spectral changes.

At pH 6.0 and $\mathrm{pH} 8.0$, instead, a slow and small phase was visible in the stopped-flow traces, which accounted for $\leq 5 \%$ of the total change in absorbance at $463 \mathrm{~nm}$ (Figure 2). At all pH values examined, the observed rate constant for the major phase was linearly dependent on the concentration of oxygen, as illustrated in Figure $3 \mathrm{~A}$ for $\mathrm{pH}$ 6.0. Instead, the small phase visible only in some of the experiments had an associated rate constant that was independent of the concentration of oxygen (Figure 3B). Flavin oxidation was assigned to the major phase seen in the stopped-flow spectrophotometer, and the associated $k_{\text {obs }}$ values were considered; the minor phase was assigned to the presence of a small fraction of enzyme not being fully functional probably due to damage ensuing during the experiment or the preparation of the enzyme. The slope of the line fitting the data in a plot of $k_{\mathrm{obs}}$ vs. [oxygen] (Figure 3A) yielded the second order rate constant $k_{\mathrm{ox}}$ for flavin oxidation, with values that were $\mathrm{pH}$-independent in the range from $\mathrm{pH} 5.0$ to $\mathrm{pH} 8.0$ (Figure 4). The $\mathrm{pH}$-independent average value was $70,000 \pm 13,000$ $\mathrm{M}^{-1} \mathrm{~s}^{-1}$ (Table 1).

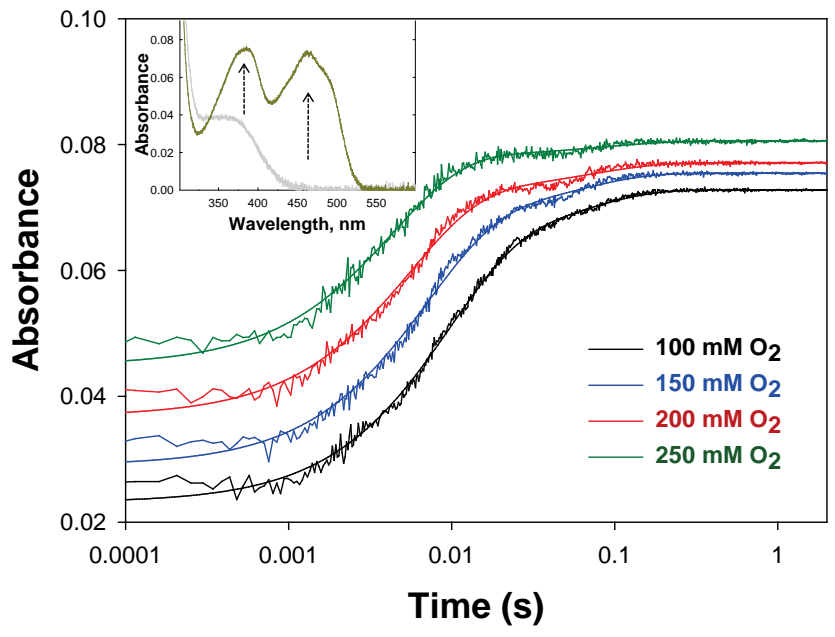

Figure 2. Time-resolved absorbance spectroscopy of the oxidation of reduced $A A O$ at varying $\mathrm{O}_{2}$ concentrations. The anaerobic enzyme was premixed with $\left[\alpha-{ }^{1}{ }_{2}\right]-p$-methoxybenzyl alcohol (0.9-fold the enzyme's concentration), allowed to age for $1 \mathrm{~s}$ and the reduced enzyme finally mixed with buffers containing various $\mathrm{O}_{2}$ concentrations (shown in legend). Traces at $463 \mathrm{~nm}$ show the two phases of the reaction, fast and slow. Solid line indicates the fit of the traces to a double-exponential process. Inset displays spectra of the initial (reduced, grey) and final (oxidized, dark yellow) flavin species, with arrows indicating the direction of the spectral changes. Conditions: enzyme ( $5 \mu \mathrm{M}$ after double mixing) in $100 \mathrm{mM}$ sodium phosphate, $\mathrm{pH} 5.0$, and $12^{\circ} \mathrm{C}$. 

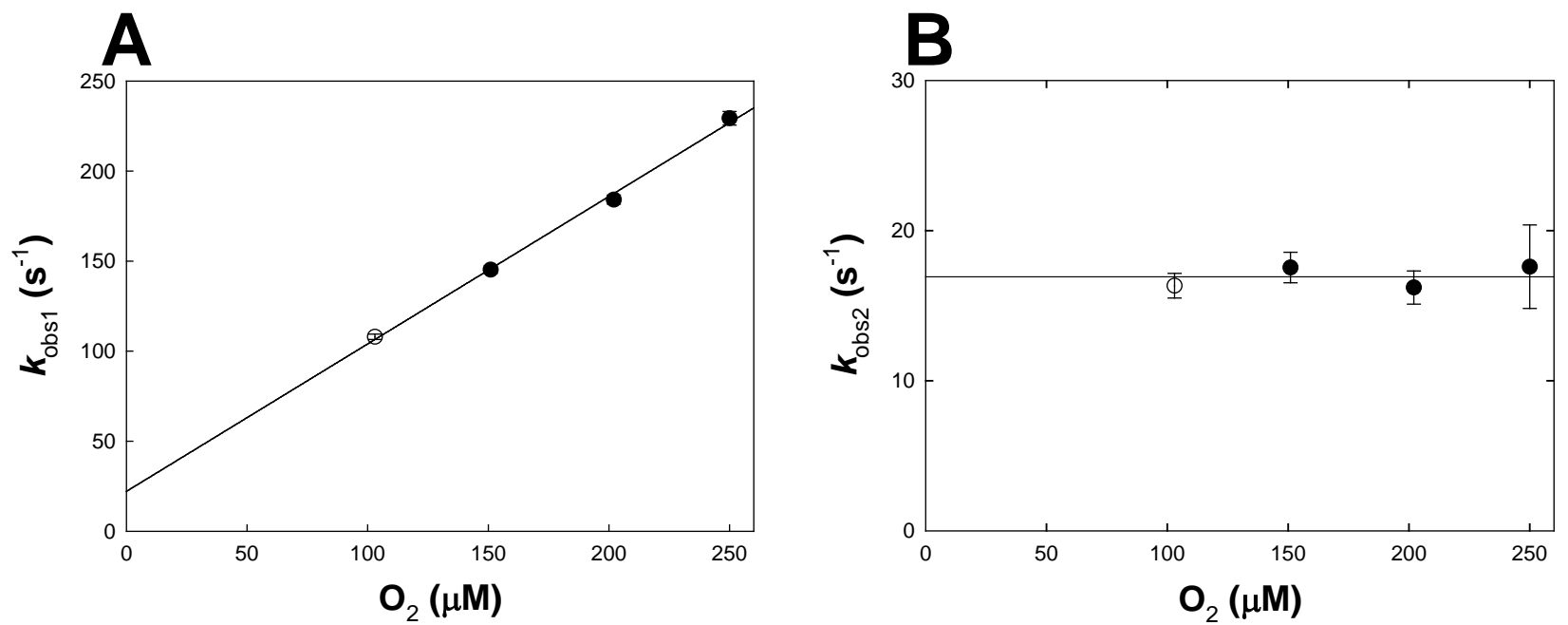

Figure 3. Plot of the observed rate constants $\left(k_{\mathrm{obs}}\right)$ for the fast $\left(k_{\mathrm{obs} 1} ; \mathrm{A}\right)$ and slow $\left(k_{\mathrm{obs} 2} ; \mathrm{B}\right)$ phases of AAO oxidation as a function of $\mathrm{O}_{2}$ concentration. The anaerobic enzyme was premixed with $\left[\alpha-{ }^{1} \mathrm{H}_{2}\right]-p$-methoxybenzyl alcohol $(0.9-$ fold the enzyme's concentration) until complete reduction, and then mixed with varying $\mathrm{O}_{2}$ concentrations (100- 
Table 1. Second-order rate constants and solvent and substrate isotope effects for the reoxidation of $A A O$ at different $\mathrm{pLS}$

\begin{tabular}{llllll}
\hline $\mathbf{p L}$ & $\boldsymbol{k}_{\text {ox }(\mathrm{H} 2 \mathrm{O})}, \mathbf{M}^{-1} \mathbf{s}^{-1}$ & $\boldsymbol{k}_{\mathrm{ox}(\mathrm{D} 2 \mathrm{O})}, \mathbf{M}^{-\mathbf{1}} \mathbf{s}^{-1}$ & $\boldsymbol{k}_{\mathrm{ox}(\mathrm{D})}, \mathbf{M}^{-\mathbf{1}} \mathbf{s}^{-1}$ & ${ }^{\mathrm{D} 20} \boldsymbol{k}_{\mathrm{ox}}$ & ${ }^{\mathrm{D}} \boldsymbol{k}_{\mathrm{ox}}$ \\
\hline 5.0 & $60,000 \pm 4,000$ & $64,000 \pm 7,000$ & & $0.9 \pm 0.1$ & \\
6.0 & $77,000 \pm 1,000$ & $86,000 \pm 5,000$ & $53,000 \pm 3,000$ & $0.9 \pm 0.1$ & $1.5 \pm 0.1$ \\
7.0 & $60,000 \pm 5,000$ & $56,000 \pm 5,000$ & & $1.1 \pm 0.1$ & \\
8.0 & $82,000 \pm 3,000$ & $67,000 \pm 5,000$ & $50,000 \pm 3,000$ & $1.2 \pm 0.1$ & $1.6 \pm 0.1$ \\
\hline Average & $\mathbf{7 0 , 0 0 0} \pm \mathbf{1 3 , 0 0 0}$ & $\mathbf{6 8 , 0 0 0} \pm \mathbf{1 3 , 0 0 0}$ & $\mathbf{5 2 , 0 0 0} \pm \mathbf{4 , 0 0 0}$ & $\mathbf{1 . 0} \pm \mathbf{0 . 3}$ & $\mathbf{1 . 5} \pm \mathbf{0 . 1}$
\end{tabular}

Data measured in $100 \mathrm{mM}$ sodium acetate pL 5.0 and 50, 30 and $25 \mathrm{mM}$ sodium phosphate pLs 6.0, 7.0 and 8.0, respectively.

To determine whether transient species could be observed in the oxidative half-reaction of AAO, the oxidation of the enzyme with molecular oxygen was monitored at $10 \mathrm{~nm}$ intervals between $300 \mathrm{~nm}$ and $540 \mathrm{~nm}$ in the single absorbance detection mode of the stopped-flow spectrophotometer, which allowed collection of $>9,000$ data points at each wavelength. The experiment was carried out at $\mathrm{pH} 5.0$ and $\mathrm{pH} 8.0$ to establish possible $\mathrm{pH}$ effects on the formation of transient species. As shown in Figure 1 for $\mathrm{pH}$ 5.0, the traces at $370 \mathrm{~nm}, 390 \mathrm{~nm}$ and $463 \mathrm{~nm}$ increased in monophasic fashion over time. The oxidation of the reduced flavin also did not show transient accumulation of any reaction intermediate(s) at pH 8.0 (Figure 2). These data are consistent with lack of observable transient species between pH 5.0 and pH 8.0 in the oxidative half-reaction catalyzed by AAO.

Solvent Effects. The second-order rate constants for flavin oxidation $k_{\text {ox }}$ were determined in buffered solutions containing $\mathrm{D}_{2} \mathrm{O}$ and the values were compared to those acquired in $\mathrm{H}_{2} \mathrm{O}$ to investigate solvent effects on the oxidative half-reaction catalyzed by AAO. As for the case of the aqueous buffers the $k_{\mathrm{ox}}$ value determined in $\mathrm{D}_{2} \mathrm{O}$ was independent of $\mathrm{pD}$ in the range from pD 5.0 to pD 8.0, with an average value of $70,000 \pm 13,000 \mathrm{M}^{-1} \mathrm{~s}^{-1}$ (Figure 4). Thus, the 
corresponding solvent kinetic isotope effect computed from the pL-independent $k_{\mathrm{ox}}$ values determined in $\mathrm{H}_{2} \mathrm{O}$ and $\mathrm{D}_{2} \mathrm{O}$ was equal to $1.03 \pm 0.27$ (Table 1). When the solvent kinetic isotope effects on the $k_{\text {ox }}$ values were computed at each $\mathrm{pL}$ values, they were also not significantly different from unity (Table 1 ).

Addition of $7.3 \%$ glycerol, which is isoviscous with $\mathrm{D}_{2} \mathrm{O}$ at $12{ }^{\circ} \mathrm{C}$, to an aqueous buffered solution at pH 6.0 resulted in no alteration of the $k_{\text {ox }}$ value which was $71,000 \pm 6,000 \mathrm{M}^{-1} \mathrm{~s}^{-1}$ with glycerol and 73,000 $\pm 3,000 \mathrm{M}^{-1} \mathrm{~s}^{-1}$ without glycerol, consistent with a negligible effect of solvent viscosity on the rate constant for flavin oxidation. These data indicate that there are no $\mathrm{pH}$, solvent viscosity or solvent kinetic isotope effects on the oxidative half-reaction catalyzed by AAO, consistent with the movements of solvent exchangeable protons not being rate-limiting for flavin oxidation in AAO.

Substrate Kinetic Isotope Effects. Previous kinetic isotope effect studies on the reductive half-reaction of AAO established that the pro- $R$ hydrogen bound to the $\alpha-C$ atom of the alcohol substrate is transferred to the N5 atom of the flavin as a hydride ion. ${ }^{15,20}$ The same $\mathrm{H}$ atom is expected to be further transferred from the N5 atom of the reduced flavin to molecular oxygen in the oxidative half-reaction, unless its wash-out from the flavin to bulk solvent is faster than the transfer to molecular oxygen.

To determine the extent of $D$ atom wash-out from the reduced flavin, AAO was anaerobically mixed in a double-mixing stopped-flow spectrophotometer with $200 \mu \mathrm{M}\left[\alpha-{ }^{2} \mathrm{H}_{2}\right]-$ $p$-methoxybenzyl alcohol, let age for different times comprised from $3 \mathrm{~s}$ to $600 \mathrm{~s}$ and mixed with atmospheric oxygen. The resulting $k_{\text {obs }}$ value for flavin oxidation at any given aging time was determined by monitoring the increase in absorbance at $463 \mathrm{~nm}$. An exponential fit of the data representing the $k_{\text {obs }}$ values as a function of the aging time yielded a value of $0.0023 \pm$ $0.0009 \mathrm{~s}^{-1}$ for the rate constant of $D$ atom wash-out from the reduced flavin, and limiting $k_{\text {obs }}$ values of $58 \pm 1 \mathrm{~s}^{-1}$ and $87 \pm 6 \mathrm{~s}^{-1}$ for the apparent rate constants for flavin oxidation extrapolated at $t=0$ and at $t=\infty$ (Figure 5). These data establish that with aging times $\leq 4 \mathrm{~s}$, [ $\alpha$ ${ }^{2} \mathrm{H}_{2}$ ]-p-methoxybenzyl alcohol can be used to probe the cleavage of the $\mathrm{N}-\mathrm{H}$ bond of the reduced flavin in the oxidative half-reaction. The ratio of the rate constant for flavin oxidation 
after the complete wash-out of the $D$ atom from the flavin to that when the $D$ atom is present on the flavin, which is equal to the apparent substrate kinetic isotope effect, is $1.5 \pm 0.1$.

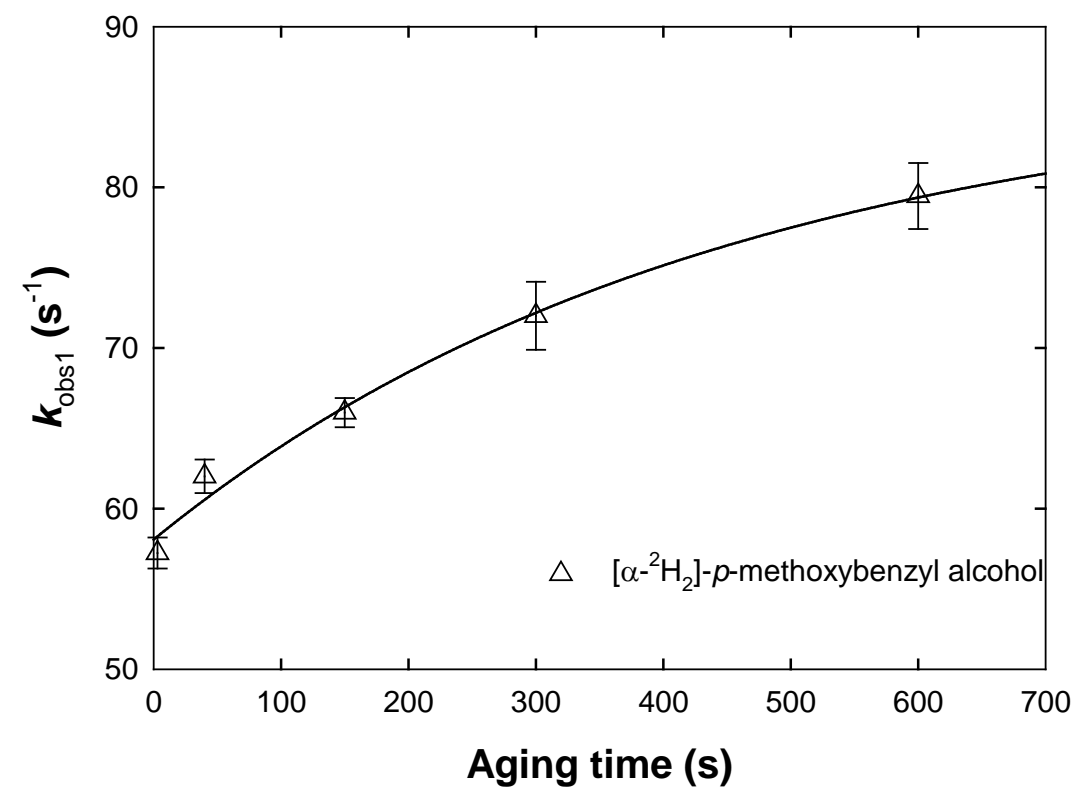

Figure 5. Deuterium wash-out from flavin N5 by exchange with protons from the protiated buffer. $k_{\text {obs }}$ dependence on the incubation time of the enzyme labeled with $\mathrm{D}$ at the $\mathrm{N} 5$ in $\mathrm{H}_{2} \mathrm{O}$ buffer. AAO was premixed and incubated with $\left[\alpha-{ }^{2} \mathrm{H}_{2}\right]-p$-methoxybenzyl alcohol (0.9-fold the enzyme's concentration) for various aging times (3-600 s), after which the reduced enzyme was mixed with $136 \mu \mathrm{M} \mathrm{O}_{2}$. Conditions: Enzyme (5 $\mu \mathrm{M}$ after double mixing) in $50 \mathrm{mM}$ sodium phosphate, $\mathrm{pH} 6.0$, at $12^{\circ} \mathrm{C}$. $k_{\text {obs }}$ values and standard deviations were estimated from triplicates and fitted to an exponential equation that yielded $y=28.7 \times\left(1-\mathrm{e}^{-0.0023 t}\right)+58.1\left(R^{2}=\right.$ 0.989).

The second-order rate constant for flavin oxidation $k_{\mathrm{ox}}$ with $\left[\alpha-{ }^{1} \mathrm{H}_{2}\right]-p$-methoxybenzyl alcohol was 77,000 $\pm 1,000 \mathrm{M}^{-1} \mathrm{~s}^{-1}$ and with $\left[\alpha-{ }^{2} \mathrm{H}_{2}\right]-p$-methoxybenzyl alcohol 53,000 $\pm 3,000 \mathrm{M}^{-}$ ${ }^{1} \mathrm{~s}^{-1}$, yielding a ${ }^{\mathrm{D}} k_{\text {ox }}$ value of $1.5 \pm 0.1$ at $\mathrm{pH} 6.0$ and $12{ }^{\circ} \mathrm{C}$. When the $\mathrm{pH}$ was changed to 8.0, similar results were observed with a ${ }^{\mathrm{D}} k_{\mathrm{ox}}$ value of $1.6 \pm 0.1$, and $k_{\mathrm{ox}}$ values of $82,000 \pm 3,000 \mathrm{M}^{-}$ ${ }^{1} \mathrm{~s}^{-1}$ and $50,000 \pm 3,000 \mathrm{M}^{-1} \mathrm{~s}^{-1}$ with the protiated and deuterated alcohol substrate, respectively. The $k_{\text {ox }}$ value was $54,000 \pm 4,000 \mathrm{M}^{-1} \mathrm{~s}^{-1}$ when $\left[\alpha-{ }^{2} \mathrm{H}_{2}\right]-p$-methoxybenzyl alcohol was used as the reducing substrate in $\mathrm{D}_{2} \mathrm{O}$ (value of the multiple KIE of $1.5 \pm 0.1$ ), further consistent with lack of solvent effects on the oxidative half-reaction of AAO. 


\section{DISCUSSION}

The catalytic cycle of AAO is composed of two half-reactions (Scheme 1). During the reductive half-reaction, FAD is reduced owing to the oxidative dehydrogenation of the alcohol substrate, which occurs in a concerted non-synchronous fashion with the transfer of one $\mathrm{H}^{-}$to flavin N5 and the abstraction of one $\mathrm{H}^{+}$by the catalytic His502. ${ }^{27}$ Subsequently, the oxidative halfreaction takes place when the enzyme donates to $\mathrm{O}_{2}$ : i) one electron; ii) one $\mathrm{H}$ atom originating from the homolytic breakage of the flavin N5-H bond; and iii) one $\mathrm{H}^{+}$from the solvent or a solvent exchangeable site, which can be the same catalytic His502 involved in the reductive half-reaction.

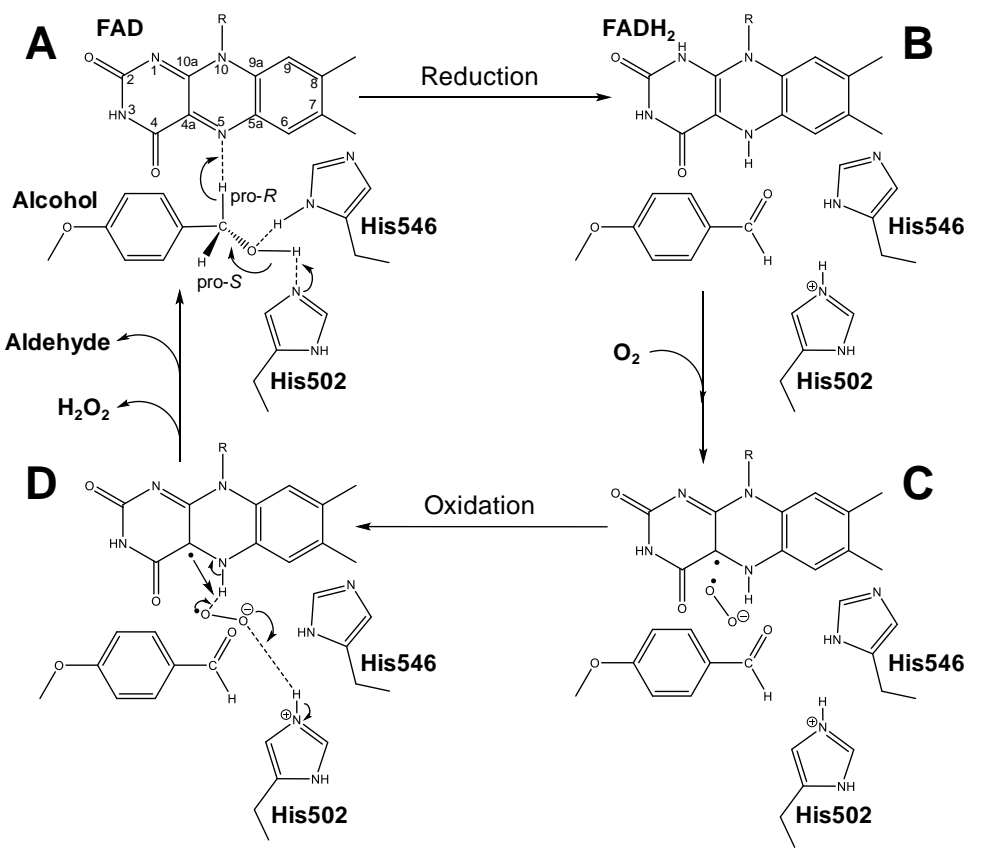

Scheme 1. $P$. eryngii AAO catalytic cycle. During AAO reduction, oxidized FAD abstracts one hydride from the alcohol pro- $R$-hydrogen assisted by His502, which abstracts the alcohol hydroxyl proton, and His546, which $\mathrm{H}-$ bonds the alcohol at the active site (A). This yields the aldehyde product, reduced FAD and protonated His502 (B). During the enzyme's oxidation, a first one-electron transfer from the flavin to $\mathrm{O}_{2}$ takes place (C), forming a protonated superoxide radical that decays rapidly and receives, in a step-wise manner, a hydrogen from flavin N5 (previously abstracted from the substrate as a hydride) and a proton (probably from a solvent exchangeable site) (D). This results in the formation of the $\mathrm{H}_{2} \mathrm{O}_{2}$ product and the two-electron oxidized FAD, without formation of any flavin detectable intermediates.

$\mathrm{O}_{2}$ reduction by $\mathrm{AAO}$ takes place without the transient accumulation of any flavin intermediate species detectable by stopped-flow spectrophotometry. Strong evidence for such mechanism derives from the thorough examination of the traces at different wavelengths 
typical of flavin intermediates, which did not reveal any intermediates at either $\mathrm{pH} 5.0$ or 8.0. Moreover, in case an intermediate - such as C4a-(hydro)peroxyflavin- was formed, it should accumulate in the presence of deuterated solvent and substrate, due to the reduced kinetic rates the isotopic substitutions cause. Nevertheless, no intermediate was detected either under such conditions. On the contrary, another oxidase, pyranose 2-oxidase (P2O), which has been considered as the missing link between oxidases and monooxygenases because of the mechanism of its oxidative half-reaction ${ }^{28}$, does stabilize a C4a-hydroperoxyflavin at $\mathrm{pHs} \mathrm{lower}$ than 8.0. When the $\mathrm{pH}$ is higher, P2O switches from this monooxygenase-like behavior to that of a typical oxidase -that is, without the stabilization of detectable intermediates-, due to the protonation state of a group with a pKa of 7.6. ${ }^{29}$

The $\mathrm{H}$ atom bound to the reduced flavin N5, which originates from the $\alpha-\mathrm{C}$ pro- $R$ position of the alcohol substrate, is subjected to slow exchange with the solvent protons. This is in agreement with the observed increase in the rate constants for flavin oxidation when the enzyme, previously reduced with deuterated substrate, is incubated in protiated buffer before being mixed with $\mathrm{O}_{2}$. Such a slow wash-out of the N5-bound atom - which shows an exchange rate of $2.3 \times 10^{-3} \mathrm{~s}^{-1}$, similar to the one reported for pyranose 2-oxidase ${ }^{12}-$ and also observed in other flavoproteins, as monooxygenases, ${ }^{30}$ is much smaller than that estimated for the exchange of $\mathrm{H}$ from the $\mathrm{N} 5$ of free $\mathrm{FMN}\left(525 \mathrm{~s}^{-1}\right)^{31}$. Such a reduced exchange rate is due to the closeness of the active site of the enzyme, which is separated from the environment by: i) a loop characteristic of the AAO family that limits the access to the active site; ${ }^{32}$ and ii) by a triad of aromatic residues (Tyr92, Phe397 and Trp501) that create a highly hydrophobic bottleneck isolating the active site from the outer environment, as crystallographic data reveal. ${ }^{33}$ This observation also indicates that the methodology used, the double-mixing stopped-flow spectrophotometry, is appropriate to study the substrate KIEs in AAO oxidation, since it permits the oxidation of the enzyme immediately after its reduction with isotopically substituted substrates.

The breakage of the bond between flavin N5 and $\mathrm{H}$ (or D) bound to it, as well as the subsequent $\mathrm{H}$ transfer to the activated $\mathrm{O}_{2}$, is a slow process that, at least partially, limits the oxidative half-reaction in AAO. Evidence for this mechanism comes from the fact that there is a 
significant effect on $k_{\mathrm{ox}}$ when employing deuterated substrates that specifically label the N5 locus with a D. It had been previously suggested that, according to QM/MM calculations, bond breakage and $\mathrm{H}$ transfer were the rate-limiting step of the oxidative half-reaction in AAO. In addition, experimental results on AAO steady-states showed that there existed a substrate KIE on the oxidative efficiency ${ }^{\mathrm{D}}\left(k_{\mathrm{cat}} / K_{\mathrm{m}(\mathrm{Ox})}\right)$ of $1.6 \pm 0.1$ at $\mathrm{pH} 6.0$, very close to the one reported here at equal $\mathrm{pH}^{19}$ Moreover, such an effect proves to be $\mathrm{pH}$ independent between $\mathrm{pH} 6.0$ and 8.0 , which means that it is not being masked by any other kinetic steps. Accordingly, steadystate kinetics carried out with AAO demonstrated that the $\mathrm{pH}$ effect on $k_{\mathrm{cat}} / K_{\mathrm{m}(\mathrm{Ox})}$ was negligible, at least until higher $\mathrm{pH}$, displaying a $\mathrm{pKa}$ of $\sim 9 .^{19}$

In contrast to the $\mathrm{H}$ transfer from flavin $\mathrm{N} 5$ to $\mathrm{O}_{2}$ discussed above, transfer of $\mathrm{H}^{+}$from the solvent or a solvent exchangeable site is a fast process that does not contribute to the ratedetermining process in AAO oxidation. This statement is evidenced by the negligible solvent isotope effect on $k_{\mathrm{ox}}$, even at the extremes of the $\mathrm{pL}$ range investigated (5.0-8.0). In this regard, previous $\mathrm{QM} / \mathrm{MM}$ studies on $\mathrm{O}_{2}$ reduction by $\mathrm{AAO}$ showed that $\mathrm{H}^{+}$transfer is a spontaneous and energetically favorable process that contributed to lowering the energy required for $\mathrm{O}_{2}$ spin inversion. ${ }^{19}$ Moreover, experimental results on AAO steady-state kinetics are further consistent with the lack of solvent $\mathrm{KIE}$, since an insignificant ${ }^{{ }_{2} \mathrm{O}}\left(k_{\mathrm{cat}} / K_{\mathrm{O}_{2}}\right)$ of $1.1 \pm 0.1$ had been found at $\mathrm{pH} 6.0 .{ }^{19}$ Absence of a solvent KIE within the $\mathrm{pH}$ range 5.0-8.0, further reinforced by the results at $\mathrm{pH} 8.0$, at which the value of the multiple KIE was not significantly different from the value of the substrate effect $(1.6 \pm 0.1)$, means that only the substrate effect contributes to limiting $k_{\text {ox }}$. Similarly, glucose oxidase, a closely-related member of the GMC superfamily, and monooxygenases show a similar pattern with negligible solvent $\mathrm{KIE}$, and consequently, fast $\mathrm{H}^{+}$ transfer. ${ }^{7,30,34}$

In the light of the above results, $\mathrm{O}_{2}$ reduction by AAO proceeds in a stepwise manner that involves various transfer processes. Evidence for this conclusion comes from the existence of, at least, one fast $\mathrm{H}^{+}$and one slower $\mathrm{H}$ transfers reactions that must be independent and, thus, non-concerted kinetic steps. According to computer simulations carried out with $\mathrm{AAO}, \mathrm{the}^{+}$ transfer would originate from the protiated His502 and would precede the N5-H bond breakage. Whether or not the $\mathrm{H}^{+}$transfer is coupled to that of the first electron, as it has been 
suggested by Wongnate and co-workers for the oxidation of pyranose oxidase, ${ }^{35}$ remains an open question in AAO. This stepwise mechanism is opposed to the transfer of $\mathrm{H}^{-}$and $\mathrm{H}^{+}$that takes place during the reductive half-reaction of the enzyme, in which both transfers are concerted, although non-synchronous. ${ }^{29}$ In contrast, the flavooxidase choline oxidase proved to transfer both the $\mathrm{H}$ and $\mathrm{H}^{+}$to $\mathrm{O}_{2}$ in a concerted manner, in the same chemical step, as arisen from the studies of multiple isotope effects on $k_{\mathrm{cat}} / K_{\mathrm{ox}} \cdot{ }^{36}$ Thus, AAO, P2O, choline oxidase, and glucose oxidase, which belong to the same GMC superfamily of flavoenzymes that oxidize alcohols, show substantial differences in the mechanistic details of their $\mathrm{O}_{2}$ reduction, perfectly exemplifying the versatility of the flavin cofactor.

In summary, KIEs, along with $\mathrm{pL}$ effects, have been employed to unravel the mechanism of AAO oxidation by dioxygen using the stopped-flow technique. Utilization of deuterated substrates gave insight into the breakage of the bond between flavin N5 atom and the $\mathrm{H}$ previously abstracted from the alcohol substrate as a hydride ion; whereas isotopically substituted solvents ruled out the presence of solvent isotope effects. These studies demonstrated that $\mathrm{O}_{2}$ reduction occurs in a non-concerted fashion, taking place in independent chemical steps. $\mathrm{H}$ transfer occurs through a slow kinetic reaction that is sensitive to isotopic substitutions, while $\mathrm{H}^{+}$transfer takes place through a fast reaction that deuterated solvents fail to slow down. Nevertheless, although this proposed mechanism for $\mathrm{O}_{2}$ reduction opens up the possibility of the stabilization of flavin intermediates, such transient species could not be detected by stopped-flow spectroscopy, as it is generally the case for oxidases.

\section{AUTHOR INFORMATION}

\section{Corresponding authors}

*GG: Department of Chemistry, Georgia State University, P.O. Box 3965, Atlanta, GA 30302-

3965, USA. E-mail: ggadda@gsu.edu. Phone: 404-413-5537. Fax: (404) 413-5505. 
*ATM: Centro de Investigaciones Biológicas, CSIC, E-28040 Madrid, Spain. E-mail:

atmartinez@cib.csic.es. Phone: +34 918373112. Fax: +34 915360432.

ORCID

Juan Carro: 0000-0002-8904-2172

Patricia Ferreira: 0000-0003-4076-6118

Angel T. Martínez: 0000-0002-1584-2863

Giovanni Gadda: 0000-0002-7508-4195

Funding

This work was supported by the European INDOX (KBBE-2013-7-613549, www.indoxproject.eu) and EnzOx2 (H2020-BBI-PPP-2015-720297, www.enzox2.eu) projects, the NOESIS project (BIO2014-56388-R) of the Spanish Ministry of Economy and Competitiveness (MINECO), and NSF project CHE-1506518.

\section{Notes}

The authors declare no competing financial interests.

\section{ACKNOWLEDGMENT}

J.C. acknowledges an FPU fellowship (FPU2012-0241) as well as an Estancias Breves FPU grant (EST14/00358) from the Spanish Ministry of Education, Culture and Sports.

\section{ABBREVIATIONS}

$\mathrm{AAO}$, aryl-alcohol oxidase; $k_{\mathrm{obs}}$, experimentally observed rate constant; ${ }^{\mathrm{D}}\left(k_{\text {cat }} / K_{\mathrm{O}_{2}}\right)$, substrate $\mathrm{KIE}$ on the catalytic efficiency of reoxidation; ${ }^{{ }_{2} \mathrm{O}}\left(k_{\mathrm{cat}} / K_{\mathrm{O}_{2}}\right)$, solvent KIE on the catalytic efficiency of reoxidation; ${ }^{\mathrm{D}} k_{\mathrm{ox}}$, substrate $\mathrm{KIE} ;{ }^{\mathrm{D} 2 \mathrm{O}} k_{\mathrm{ox}}$ solvent $\mathrm{KIE} ; k_{\text {rev }}$, reverse constant; $\mathrm{GMC}$, glucosemethanol-choline oxidase/dehydrogenase superfamily; KIE, kinetic isotope effect; P2O, pyranose 2-oxidase; PMT, photomultiplier; $\mathrm{pL}$, pH or PD. 


\section{REFERENCES}

(1) Romero, E., Gómez Castellanos, J. R., Gadda, G., Fraaije, M. W., and Mattevi, A. (2018) Same substrate, many reactions: Oxygen activation in flavoenzymes. Chem. Rev.

(2) Sawyer, D. T. (1988) The redox thermodynamics for dioxygen species $\left(\mathrm{O}_{2}, \mathrm{O}_{2}{ }^{-}\right.$, $\mathrm{HOO}$, $\left.\mathrm{HOOH}, \mathrm{HOO}^{-}\right)$an monooxygen species $\left(\mathrm{O}, \mathrm{O}^{-}, \mathrm{OH}\right.$, and $\left.{ }^{\circ} \mathrm{OH}\right)$ in water and aprotic solvents. Basic Life Sci. 49, 11-20.

(3) Malmström, B. G. (1982) Enzymology of oxygen. Annu. Rev. Biochem. 51, 21-59.

(4) Mattevi, A. (2006) To be or not to be an oxidase: challenging the oxygen reactivity of flavoenzymes. Trends Biochem. Sci. 31, 276-283.

(5) Massey, V. (1994) Activation of molecular oxygen by flavins and flavoproteins. J. Biol. Chem. 269, $22459-22462$.

(6) Pennati, A., and Gadda, G. (2011) Stabilization of an intermediate in the oxidative halfreaction of human liver glycolate oxidase. Biochemistry 50, 1-3.

(7) Roth, J. P., and Klinman, J. P. (2003) Catalysis of electron transfer during activation of $\mathrm{O}_{2}$ by the flavoprotein glucose oxidase. Proc. Natl. Acad. Sci. USA 100, 62-67.

(8) Gadda, G. (2012) Oxygen activation in flavoprotein oxidases: The importance of being positive. Biochemistry 51, 2662-2669.

(9) Klinman, J. P. (2007) How do enzymes activate oxygen without inactivating themselves? Account. Chem. Res. 40, 325-333.

(10) Ghisla, S., and Massey, V. (1989) Mechanisms of flavoprotein-catalyzed reactions. Eur. J. Biochem. 181, 1-17.

(11) Sucharitakul, J., Prongjit, M., Haltrich, D., and Chaiyen, P. (2008) Detection of a C4ahydroperoxyflavin intermediate in the reaction of a flavoprotein oxidase. Biochemistry 47, 8485-8490.

(12) Sucharitakul, J., Wongnate, T., and Chaiyen, P. (2011) Hydrogen peroxide elimination from C4a-hydroperoxyflavin in a flavoprotein oxidase occurs through a single proton transfer from flavin N5 to a peroxide leaving group. J. Biol. Chem. 286, 16900-16909.

(13) Gannavaram, S., and Gadda, G. (2013) Relative timing of hydrogen and proton transfers in the reaction of flavin oxidation catalyzed by choline oxidase. Biochemistry 52, 1221-1226.

(14) Ferreira, P., Hernández-Ortega, A., Lucas, F., Carro, J., Herguedas, B., Borrelli, K. W., Guallar, V., Martínez, A. T., and Medina, M. (2015) Aromatic stacking interactions govern catalysis in aryl-alcohol oxidase. FEBS J. 282, 3091-3106.

(15) Ferreira, P., Hernández-Ortega, A., Herguedas, B., Martínez, A. T., and Medina, M. (2009) Aryl-alcohol oxidase involved in lignin degradation: A mechanistic study based on steady and pre-steady state kinetics and primary and solvent isotope effects with two different alcohol substrates. J. Biol. Chem. 284, 24840-24847.

(16) Hernández-Ortega, A., Ferreira, P., Merino, P., Medina, M., Guallar, V., and Martínez, A. T. (2012) Stereoselective hydride transfer by aryl-alcohol oxidase, a member of the GMC superfamily. ChemBioChem 13, 427-435.

(17) Ferreira, P., Hernández-Ortega, A., Herguedas, B., Rencoret, J., Gutiérrez, A., Martínez, M. J., Jiménez-Barbero, J., Medina, M., and Martínez, A. T. (2010) Kinetic and chemical 
characterization of aldehyde oxidation by fungal aryl-alcohol oxidase. Biochem. J. 425, 585-593.

(18) Hernández-Ortega, A., Lucas, F., Ferreira, P., Medina, M., Guallar, V., and Martínez, A. T. (2011) Modulating $\mathrm{O}_{2}$ reactivity in a fungal flavoenzyme: Involvement of aryl-alcohol oxidase Phe-501 contiguous to catalytic histidine. J. Biol. Chem. 286, 41105-41114.

(19) Hernández-Ortega, A., Lucas, F., Ferreira, P., Medina, M., Guallar, V., and Martínez, A. T. (2012) Role of active site histidines in the two half-reactions of the aryl-alcohol oxidase catalytic cycle. Biochemistry 51, 6595-6608.

(20) Carro, J., Matínez-Júlvez, M., Medina, M., Martínez, A. T., and Ferreira, P. (2017) Protein dynamics promote hydride tunnelling in substrate oxidation by aryl-alcohol oxidase. Phys. Chem. Chem. Phys. 19, 28666-28675.

(21) Guillén, F., Martínez, A. T., Martínez, M. J., and Evans, C. S. (1994) Hydrogen peroxideproducing system of Pleurotus eryngii involving the extracellular enzyme aryl-alcohol oxidase. Appl. Microbiol. Biotechnol. 41, 465-470.

(22) Carro, J., Ferreira, P., Rodríguez, L., Prieto, A., Serrano, A., Balcells, B., Ardá, A., JiménezBarbero, J., Gutiérrez, A., Ullrich, R., Hofrichter, M., and Martínez, A. T. (2015) 5Hydroxymethylfurfural conversion by fungal aryl-alcohol oxidase and unspecific peroxygenase. FEBS J. 282, 3218-3229.

(23) Leskovac, V., Trivic, S., Wohlfahrt, G., Kandrac, J., and Pericin, D. (2005) Glucose oxidase from Aspergillus niger: The mechanism of action with molecular oxygen, quinones, and one-electron acceptors. Int. J. Biochem. Cell Biol. 37, 731-750.

(24) Mathieu, Y., Piumi, F., Valli, R., Aramburu, J. C., Ferreira, P., Faulds, C. B., and Record, E. (2016) Activities of secreted aryl alcohol quinone oxidoreductases from Pycnoporus cinnabarinus provide insights into fungal degradation of plant biomass. Appl. Environ. Microbiol. 82, 2411-2423.

(25) Ruiz-Dueñas, F. J., Ferreira, P., Martínez, M. J., and Martínez, A. T. (2006) In vitro activation, purification, and characterization of Escherichia coli expressed aryl-alcohol oxidase, a unique H2O2-producing enzyme. Protein Express. Purif. 45, 191-199.

(26) Cheng, N. S. (2008) Formula for the viscosity of a glycerol-water mixture. Ind. Eng. Chem. Res. 47, 3285-3288.

(27) Hernández-Ortega, A., Borrelli, K., Ferreira, P., Medina, M., Martínez, A. T., and Guallar, V. (2011) Substrate diffusion and oxidation in GMC oxidoreductases: An experimental and computational study on fungal aryl-alcohol oxidase. Biochem. J. 436, 341-350.

(28) Chaiyen, P., Fraaije, M. W., and Mattevi, A. (2012) The enigmatic reaction of flavins with oxygen. Trends in Biochemical Sciences 37, 373-380.

(29) Prongit, M., Sucharitakul, J., Palfey, B. A., and Chaiyen, P. (2013) Oxidation mode of pyranose 2-oxidase is controlled by $\mathrm{pH}$. Biochemistry 52, 1437-1445.

(30) Robinson, R., Badieyan, S., and Sobrado, P. (2013) C4a-Hydroperoxyflavin formation in Nhydroxylating flavin monooxygenases is mediated by the 2'-OH of the nicotinamide ribose of NADP ${ }^{+}$. Biochemistry 52, 9089-9091.

(31) Macheroux, P., Ghisla, S., Sanner, C., Ruterjans, H., and Muller, F. (2005) Reduced flavin: NMR investigation of N5-H exchange mechanism, estimation of ionisation constants and assessment of properties as biological catalyst. BMC Biochem. 6, 26. 
(32) Ferreira, P., Carro, J., Serrano, A., and Martínez, A. T. (2015) A survey of genes encoding $\mathrm{H}_{2} \mathrm{O}_{2}$-producing GMC oxidoreductases in 10 Polyporales genomes. Mycologia 107, 11051119.

(33) Fernández, I. S., Ruiz-Dueñas, F. J., Santillana, E., Ferreira, P., Martínez, M. J., Martínez, A. T., and Romero, A. (2009) Novel structural features in the GMC family of oxidoreductases revealed by the crystal structure of fungal aryl-alcohol oxidase. Acta Crystallogr. D. Biol. Crystallogr. 65, 1196-1205.

(34) Bright, H. J., and Gibson, Q. H. (1967) The oxidation of 1-deuterated glucose by glucose oxidase. J. Biol. Chem. 242, 994-1003.

(35) Wongnate, T., Surawatanawong, P., Visitsatthawong, S., Sucharitakul, J., Scrutton, N. S., and Chaiyen, P. (2014) Proton-coupled electron transfer and adduct configuration are important for C4a-hydroperoxyflavin formation and stabilization in a flavoenzyme. J. Am. Chem. Soc. 136, 241-253.

(36) Gannavaram, S., and Gadda, G. (2013) Relative timing of hydrogen and proton transfers in the reaction of flavin oxidation catalyzed by choline oxidase. Biochemistry 52, 1221-1226. 
For Table of Contents Use Only

\title{
Stepwise Hydrogen Atom and Proton Transfers in Dioxygen Reduction by Aryl-Alcohol Oxidase
}

\author{
Juan Carro, Patricia Ferreira, Angel T. Martínez and Giovanni Gadda
}

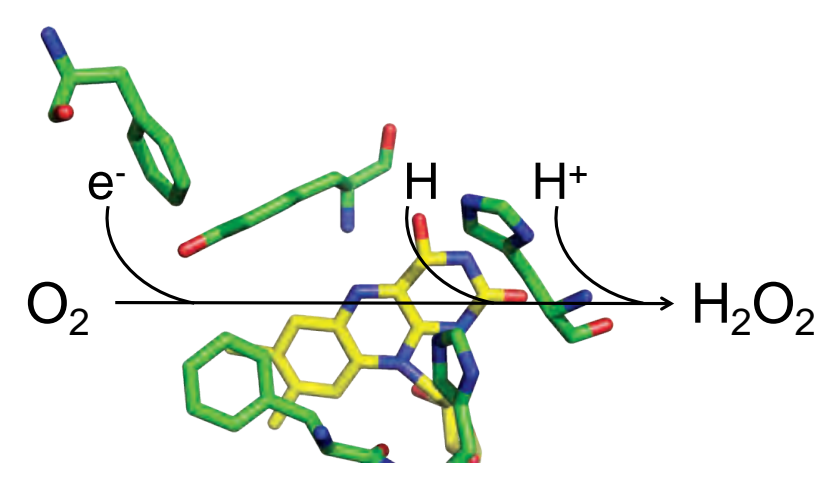

